\title{
MAPEAMENTO DE PROCESSOS EM UMA EMPRESA DE EQUIPAMENTOS E SERVIÇOS OFFHORE
}

\author{
Fabrício Alves do Nascimento \\ Universidade Candido Mendes \\ Campos dos Goytacazes, RJ \\ fabricionasciment@yahoo.com.br
}

\begin{abstract}
RESUMO
O presente estudo tem por objetivo demonstrar a aplicabilidade da técnica do Mapeamento de Processos para fins de avaliação de cada etapa desempenhada pela linha de serviços de inspeção e reparo em tubos de perfuração, no qual a ferramenta utilizada foi a (Suppliers Inputs Process Outputs Costumers) SIPOC. Para isso realizou-se um mapeamento no processo produtivo de uma empresa prestadora de serviços, atuante no ramo offshore na bacia de Campos, demonstrando as particularidades, dificuldades, tecnologias e procedimentos aplicados em cada etapa da produção. Com isso, os gestores visualizariam de forma clara e simples as etapas do negócio, no intuito de melhorar a totalidade dos processos que juntos resultam no produto final: bens e serviços. Para atingir o sucesso é necessário que ações de uma empresa gerem produtos e serviços de qualidade, pois aumentam as possibilidades da organização de se estabilizar no mercado, obter mais lucro e maior tempo de vida. Assim se faz importante utilizar-se de recursos que apontem os erros nos processos produtivos, diminua ou esclareça a complexidade das operações e reduza custos, aumentando a performance dos processos e trazendo mais conhecimento do próprio negócio.
\end{abstract}

Palavra-chave: Serviços; Mapeamento de Processos; SIPOC.

\begin{abstract}
The present study aims to demonstrate the applicability of the Process Mapping technique for the purpose of evaluating each step performed by the line of inspection and repair services in drilling pipes, in which the tool used was the SIPOC. For this purpose, a mapping was carried out in the production process of a service company operating in the offshore industry in the Campos basin, demonstrating the particularities, difficulties, technologies and procedures applied at each stage of production. In this way, the managers would visualize in a clear and simple way the steps of the business, in order to improve the totality of the processes that together result in the final product: goods and services. To achieve success, it is necessary that a company's actions generate quality products and services because they increase the organization's chances of stabilizing in the market, obtaining more profit and a longer life. Thus, it is important to use resources that point out errors in production processes, reduce or clarify the complexity of operations and reduce costs, increasing process performance and bringing more knowledge of the business itself.
\end{abstract}

Keywords: Services; Mapping of Processes; SIPOC. 


\section{INTRODUÇÃO}

Nesta pesquisa foram realizados estudos referentes a uma determinada linha de serviços da organização, em uma empresa do ramo offshore sediada no município de Macaé, interior do Estado do Rio de Janeiro, localizada na bacia petrolífera de Campos. Com a utilização da ferramenta Supplier, Input, Process, Output, Customer (SIPOC), demonstrando seu funcionamento com as suas técnicas no processo investigado para os serviços de inspeção e reparo em tubos de perfuração.

Considerando o caráter da área de concentração, a Gerência da Produção e os interesses e objetivos da linha de pesquisa, que vem desenvolvendo, entre outras ações, pesquisas e práticas que se relacionam com a produção e o trabalho, possíveis a se aplicar na organização, percebemos a oportunidade de realizar pesquisas e desenvolver projetos que colaborem para o crescimento e solidez da empresa em questão. Em um mercado financeiro competitivo as organizações precisam ser cada vez mais efetivas nas atividades e nos processos exercidos por ela, é de suma importância que os mesmos sejam conhecidos por todos os colaboradores desta instituição, portanto se faz necessário uma melhor visualização do processo organizacional, isso se dá através da prática de ferramenta no gerenciamento de processos.

O Mapeamento de processos utilizado através da ferramenta SIPOC possibilita a comunicação entre as partes interessadas, a descoberta de informações relevantes ao processo e os recursos para um melhor entendimento das atividades que possam auxiliar na melhoria e eficácia das funções exercidas no ambiente empresarial, onde resulta a produção de bens e serviços. Mapear os processos ajuda a empresa a enxergar com clareza pontos fortes e fragilidades dentro de cada etapa da produção, entender a complexidade e perceber a particularidades de cada setor, auxilia na análise dos custos que podem ser reduzidos, diminui erros e obtém maior controle de documentos e ações da organização.

Utilizando a ferramenta de Mapeamento dos Processos a problemática da pesquisa se desenvolverá na aplicabilidade da técnica a fim de otimizar o processo produtivo da empresa e aumentar a eficácia e eficiência destes. Entendendo as limitações da pesquisa, pois se tratando de um estudo de caso não há como prever o comportamento dos setores envolvidos considerando que os dados foram concedidos de uma empresa real atuante no mercado de Petróleo \& Gás da bacia petrolífera de Campos.

\section{REFERENCIANDO PROCESSOS}

"Um processo é um grupo de atividades realizadas numa sequência lógica com o objetivo de produzir um bem ou um serviço que tem valor para um grupo específico de clientes" (HAMMER; CHAMPY, 1994, apud GONÇALVES, 2000, p.2). As organizações são compostas por uma cadeia de processos que trabalham em conjunto para trazer um determinado resultado. O bom andamento destes processos produz efeitos positivos nos resultados, que serão entregues ao cliente. Isso significa que os processos refletem como a empresa funciona e são eles os responsáveis pela criação do valor na perspectiva do cliente.

"Um programa de melhoria da qualidade no âmbito da companhia supõe que todos os empregados têm a capacidade de motivação própria e pensamento criativo. Aos empregados é dado suporte, e suas ideias são solicitadas em um ambiente de mútuo respeito". (Fitzsimmons, Fitzsimmons 2000, p.393). O planejamento estratégico visa a melhoria 
contínua dos processos ocorridos na empresa e para isso se utiliza de ferramentas de gestão tal como o Mapeamento de Processos.

O Mapeamento de Processos é uma técnica utilizada para a compreensão da forma como um trabalho flui dentro de uma organização ou sistema. Esta técnica utiliza-se de diagramas de fluxo de processos que fornecem detalhes sequenciais de como uma tarefa é executada. Ele cria um vocabulário que permite um estudo de como o atual processo funciona, identificando problemas, limitações e oportunidades de melhoria através de diagramas de fluxo de processo" (O’BRIEN et al, 1994).

Com o mapeamento dos processos, utilizando fluxogramas, a relevância de uma melhor compreensão da atividade analisada aumenta de certa forma o nível de entendimento de cada etapa. Possibilitando assim a identificação de certos entraves que prejudicam o bom andamento da produtividade em questão, com isso sendo possível estudar propostas de melhorias onde foram encontrados determinados gargalos.

Segundo Slack, Chambers e Johnston (2002), a partir da perspectiva da gestão por processos, podemos definir processos (de negócio) como a coleção das contribuições que cada microoperação realizada na organização faz, de modo a satisfazer as necessidades dos consumidores e que, geralmente, cruzam os limites organizacionais convencionais.

Os processos bem definidos contribuem para os gestores elaborarem projetos sólidos, estruturados e planejados, pois cada etapa da produção do serviço a ser executado necessita de procedimentos implementados com devida rigorosidade, visando o ponto principal a atingir que são os resultados.

De acordo com De Sordi (2012), uma organização administrada por processos passa a operar não de forma hierárquica, mas por meios de estruturas definidas com meta nos objetivos dos negócios. Com isso, compreende melhor o funcionamento de cada etapa executada pelos colaboradores e o seu devido fluxo, com um objetivo claro e abrangente, sendo possível atingir um resultado satisfatório.

Conforme o relato de Martinhão Filho e Souza (2006), a ferramenta SIPOC é utilizada como técnica, com o objetivo de levantar pontos relevantes para a melhoria do processo, demonstrando o conhecimento por completo de cada etapa, com isso identificando as não-conformidades existentes. A ferramenta possibilita uma visão geral de todo o processo: Fornecedor, Entrada, Processo, Saída e Cliente.

Figura 1: Ferramenta SIPOC (Suppliers, Inputs, Process, Outputs, Customers)

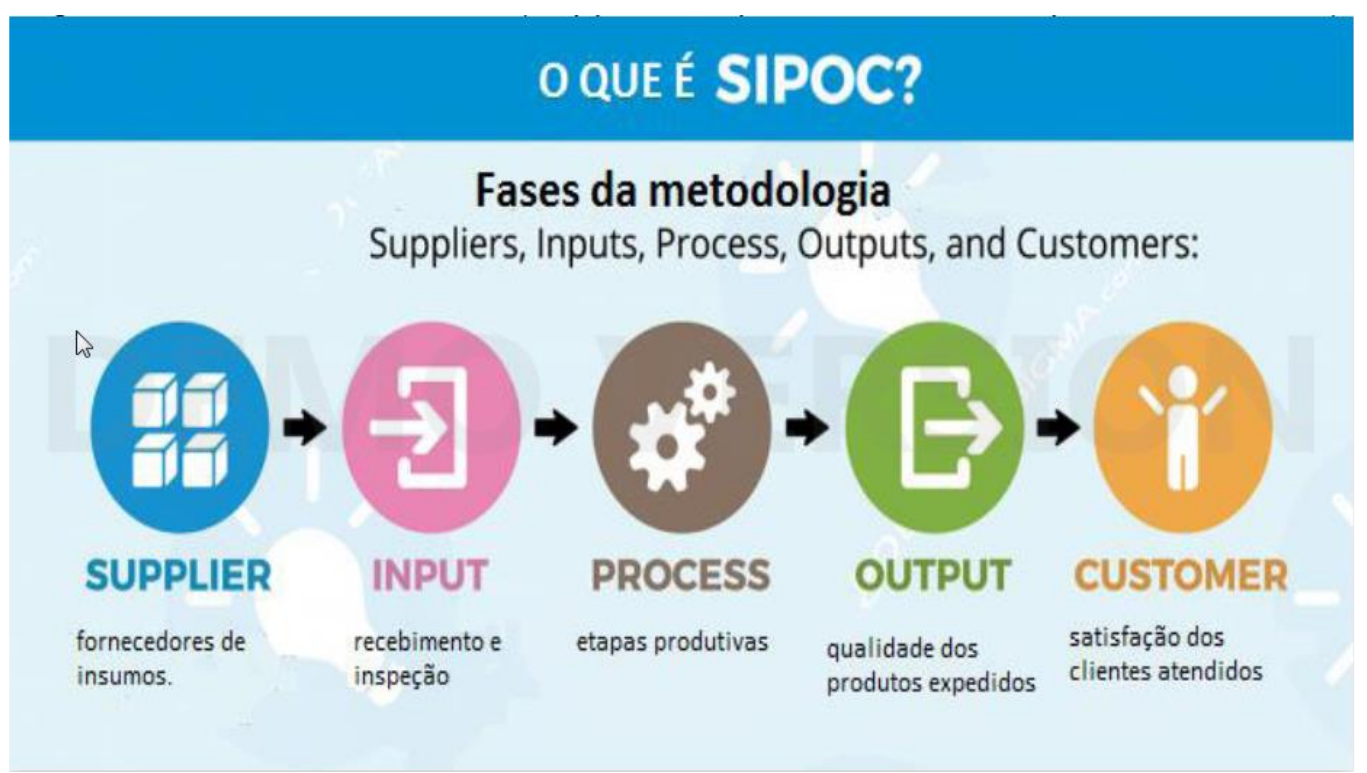

Fonte: SWAN (2018) 
De acordo com George (2003), o SIPOC é definido em sua forma no início do projeto, porém com seu andamento ocorrem as reestruturações e mudanças no projeto, causando efeitos de melhoria. No diagrama de SIPOC contém as seguintes informações:

- Fornecedores: entidades que disponibilizaram as entradas do processo para ser trabalhado.

- Entrada: materiais que entram no processo e que sofrem modificações e serão transformados em saída.

- Processo: são etapas que agregam valor, quanto as que não agregam valor, identificando o processo analisado no SIPOC.

- Saída: produto ou serviço enviado ao cliente após sofrer modificações no processo.

- Clientes: o cliente externo recebe o resultado do processo.

O SIPOC consiste em um mapa de elevado nível, sendo possível visualizar de forma geral todos os componentes participantes do processo. Tal ferramenta é de suma importância quando as informações não estiverem claras: quem são os clientes do processo, quais são as exigências do cliente, de onde vem as contribuições do processo e que especificações são contribuídas (BRADY, 2013).

Com a utilização da ferramenta SIPOC, é possível obter um controle maior de entradas e saídas durante o processo e, com isso, evitar falhas no resultado final, sendo possível levantar indicadores de eficiência e eficácia (PETENATE, 2012). De tal modo a SIPOC permite visualizar tais procedimentos implantados em uma linha de produção, possibilitando sua identificação, se em alguma etapa do processo necessita sofrer modificações, para fins de melhoria no mapeamento de processos.

\section{METODOLOGIA}

Esta pesquisa se caracteriza como estudo de caso, no qual foram obtidas as informações necessárias por meio de um questionário aplicado a um funcionário da organização. Do ponto de vista da abordagem, a pesquisa empregada nesta pesquisa se dá de forma qualitativa. Richardson (1999, p. 80) faz a seguinte colocação: “[...] os estudos que empregam uma metodologia qualitativa podem descrever a complexidade de determinado problema, analisar a interação de certas variáveis, compreender e classificar processos dinâmicos vividos por grupos sociais".

O estudo de caso é um dos mais recomendáveis, quando a questão que rege a proposta da pesquisa gira em torno da observação de procedimentos já utilizados para a construção de novas formas de atuação ou melhor aproveitamento de processos existentes tratando como elos operacionais que precisam ser traçados ao longo do tempo. Escolheu-se o método qualitativo, pois, além de se tratar de uma empresa específica do município de Macaé, ele permite que o entrevistado possa expor, com liberdade, sua opinião, sua dificuldade e sugestões para que o processo seja facilitado. Os dados foram coletados por meio de entrevista com coordenador responsável pelo setor operacional da linha analisada.

\subsection{Caracterização da empresa}

A empresa investigada está localizada no munícipio de Macaé, interior do Estado do Rio de Janeiro, atuante no ramo de exploração da indústria de petróleo e gás. A organização também presta serviços de cadeia de suprimentos através da sua rede de centros de 
distribuição e serviços, sendo a linha principal inspeção e reparos em tubos, que estão localizados próximos às principais atividades de perfuração e produção ao redor do mundo.

Foi elaborado a matriz SIPOC, conforme demonstrado no quadro 1, onde foram informados os indicadores existentes: fornecedores, entradas, processo, saídas e clientes. Essa ferramenta contribuiu para uma melhor identificação de cada processo a ser analisado e estruturado para uma melhor visualização do processo.

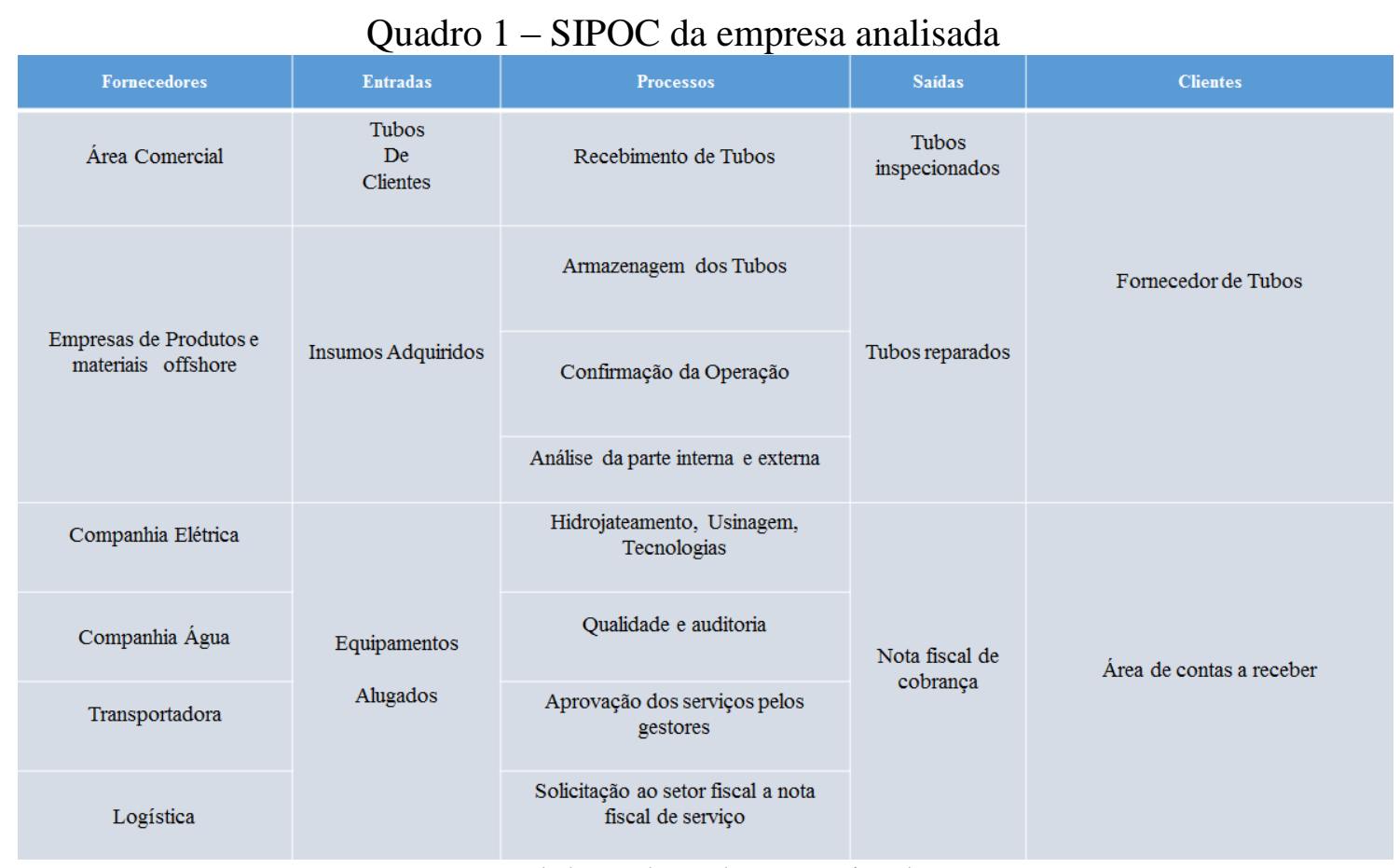

Fonte: elaborado pelo pesquisador.

Após a elaboração do SIPOC, podemos mencionar com detalhes as etapas dos processos em questão. Para que a ferramenta seja iniciada faz-se necessário que a área comercial informe um projeto fechado com um determinado cliente, assim começará os trâmites envolvendo diversos colaboradores da operação que informaram a necessidade de adquirirem novos produtos para o bom funcionamento do serviço. Ligado a primeira etapa fornecedores temos os recursos principais a serem utilizados como compra com empresas de materiais offshore, a logística, transportadora, companhia de água e luz elétrica que é o fator principal para o funcionamento dos equipamentos.

A segunda etapa do processo envolve as Entradas dos produtos, que são o recebimento dos tubos que o cliente enviou junto com a ordem de compra para inspeção e reparo com as devidas particularidades foram mencionadas durante o fechamento do projeto. Neste processo ocorrem também o recebimento dos insumos adquiridos pelos fornecedores, e a entrada de diversos equipamentos alugados para sua utilização na operação da produção.

$\mathrm{Na}$ terceira parte os processos ocorrem a partir do momento em que os tubos encaminhados pelos clientes se encontram dentro da empresa, logo os mesmos são armazenados e registrados no sistema da empresa e do governo informando a confirmação da operação através de sua nota fiscal recebida. Sendo assim começam a execução dos serviços nos materiais de acordo com o projeto informado pelo cliente, passando pelo processo de inspeção ou reparo, realizando assim um hidro jateamento tanto na parte interna quanto externa. São feitas usinagens para as devidas conexões de inspeções tubulares, para produção 
de reparos e revestimentos dos seguintes tipos de tubos: Drill pipe, Drill Collar, Heavy Weight e Crossovers. Nesta etapa os tubos são inspecionados por vários equipamentos tecnológico para os de reparos são usados os equipamentos: Torno CNC e Programação Assistida, já para os de inspeção são utilizadas: partículas magnéticas, Ultrassom Utmaax, Inspeção Visual e Desempeno de Tubos. Depois da finalização dos serviços, o setor de qualidade e auditoria revisam os materiais prontos e elaboram um laudo informando o resultado do processo, em seguida passam pela aprovação dos gestores responsáveis pelas atividades. Após aprovado e finalizados os serviços são gerados relatórios para fins de documentação e solicitado ao setor fiscal a nota fiscal de cobrança.

No processo de saídas são encaminhados os tubos prontos inspecionados e reparados para os clientes juntamente com a nota fiscal eletrônica de cobrança. O cliente recebe os tubos conforme programado no projeto e no prazo de entrega acordado, em seguida temos o processo de contas a receber que a partir da data de emissão da nota fiscal, no prazo de 30 dias controla o recebimento do serviço prestado finalizando o processo.

Durante determinado período de coleta das informações relevantes aos pontos de cada processo, identificou-se uma etapa que estava impactando o prazo de entrega dos materiais para o cliente. $\mathrm{O}$ fato ocorria no momento em que a linha de produção iniciava suas atividades com os materiais recebidos, acabavam encontrando diversas irregularidades da situação atual dos tubos que foram acordados com o cliente para execução do projeto, tais materiais chegavam nos seguintes estados: trocados, trincados, empenados, com a rosca danificada ou até mesmo amassado. Diante de tais fatos ocorridos o responsável pela operação entrava em contato com o cliente e informava os problemas encontrados nos materiais recebidos, buscando a melhor forma de resolver a situação ocorrida.

\section{RESULTADO DA PESQUISA}

Com a linha de estudo investigada, foram coletados dados enriquecedores que contribuíram para o desenvolvimento da pesquisa, o entrevistado da organização informou as respostas necessárias para o estudo, no qual a linha estudada possui 1 gerente, 2 coordenadores e 32 funcionários que executam diretamente o passo a passo da produtividade. A área comercial influencia para o desenvolvimento do serviço, que são os tramites feitos no projeto com os clientes, o fluxo de entrada de compras materiais ocorre pelo setor de compras através de requisições feitas pelos solicitantes, tendo os fatores importantes na execução das atividades: energia, água, logística e transportadora. $\mathrm{O}$ serviço de inspeção e reparo são desenvolvidos com hidro jatos, ultrassom, partículas magnéticas e usinagem, com isso realizando o serviço dentro do prazo acordado com o cliente o serviço de inspeção em 50 tubos em 2 dias.

Verificamos que determinada etapa do processo estava ocorrendo atrasos na entrega do projeto e causando diversos impactos nos resultados. Com isso foi estudado uma proposta de melhoria para resolução do problema neste processo, para fins de diminuir erros durante as fases do projeto, que foram elaborados da seguinte maneira. O colaborador responsável por receber os materiais, tem que checar os seguintes itens: conferência da embalagem, condições dos produtos, danificações, origem, lote de fabricação, quantidade, tipo de material, data, marcação e certificado de qualidade.

Foi elaborado um formulário para melhor implementação e controle dos materiais conforme a figura abaixo, devendo seguir os seguintes requisitos para o devido recebimento dos tubos. 
Quadro 2 - Formulário

CONTROLE DE RECEBIMENTO DOS MATERIAIS

\begin{tabular}{|c|c|c|}
\hline \multirow{2}{*}{ Logomarca da Empresa } & Empresa & \multirow{2}{*}{$\mathrm{N}^{\mathrm{o}}$} \\
\hline & Endereço: & \\
\hline \multicolumn{3}{|l|}{ Nome do Projeto } \\
\hline Data chegada do material & \multicolumn{2}{|c|}{1} \\
\hline \multicolumn{3}{|l|}{ Cliente } \\
\hline \multicolumn{3}{|l|}{ Descrição do Material } \\
\hline \multicolumn{3}{|l|}{ Quantidade } \\
\hline Valor Unitário & Valor Total & $\mathrm{R} \$$ \\
\hline \multicolumn{3}{|l|}{ Nota Fiscal N ${ }^{\circ}$} \\
\hline Data de emissão & \multicolumn{2}{|c|}{11} \\
\hline \multicolumn{3}{|l|}{ Certificados } \\
\hline \multicolumn{3}{|l|}{ Cotação No } \\
\hline $\mathrm{PO}$ & \multicolumn{2}{|c|}{ ( ) SIM ( ) NÃO N ${ }^{\circ}$} \\
\hline CONDIÇÕES DOS MATERIAIS & \multicolumn{2}{|c|}{ SITUAÇÃO } \\
\hline Embalagem & \multicolumn{2}{|c|}{ ( ) Boa ( ) Ruim } \\
\hline Estado dos produtos & \multicolumn{2}{|c|}{ ( ) Bom ( ) Ruim } \\
\hline \multicolumn{3}{|l|}{ Origem } \\
\hline \multicolumn{3}{|l|}{ Lote } \\
\hline Inconformidade & \multicolumn{2}{|l|}{ ( ) $\operatorname{Sim}($ ) Não } \\
\hline \multicolumn{3}{|l|}{ Observações: } \\
\hline \multicolumn{3}{|c|}{ Aprovado recebimento: ( ) Sim ( ) Não } \\
\hline \multicolumn{3}{|l|}{ Recebido por: } \\
\hline Nome Completo e Car & abo & Assinatura \\
\hline
\end{tabular}

Fonte: elaborado pelo pesquisador. 
Verificamos que a pesquisa, feita por meio de questionário, e logo em seguida implementada dentro da ferramenta SIPOC, possibilitou uma melhor visualização e compreensão do funcionamento de todas as etapas do processo de inspeção e reparo em tubos de perfuração. Durante o estudo analisado e gerado pelo método SIPOC, foi possível identificar um impacto em determinado momento do processo, gerando efeitos negativos nos resultados. Com isso foi elaborado um novo procedimento para inserção no processo, por meio de um formulário de controle, devendo ser seguidos todos os requisitos necessários gerando efeitos positivos no recebimento dos tubos. Com a utilização do formulário durante o recebimento dos tubos é possível evitar erros durante a realização do serviço executado dentro da organização. Com isso fazendo toda a conferência e seguindo as regras de conformidade e validação da qualidade dos materiais recebidos, referente ao cumprimento de todos os itens disponibilizados no formulário. Sendo possível obter resultados satisfatórios e garantindo a fidelidade com os clientes, demonstrando assim competência e qualidade desde o início do processo do serviço até a sua entrega final sem ocorrência de erros e transtornos de devolução de material.

\section{CONSIDERAÇÕES FINAIS}

Por meio desta pesquisa foi possível compreender o funcionamento da aplicação da ferramenta SIPOC e seus efeitos resultantes no decorrer de cada etapa dos processos. Com as respostas do questionário aplicado foi possível alcançar dados qualitativos para elaboração do quadro SIPOC e seus efeitos significativos para uma melhor visualização do funcionamento da linha de serviços de inspeção e reparo.

O mapeamento de processos através da ferramenta SIPOC demonstrou que em determinada parte do processo necessita de um novo procedimento, no qual foi elaborado no artigo, para fins de melhorar o fluxo das atividades diárias.

A construção deste artigo foi de suma importância, pois possibilitou a aplicação da ferramenta SIPOC em um estudo de caso real. Com isso sendo possível identificar falhas nos processos e gerar uma opção para resolução do problema. Podemos ressaltar que para uma organização se manter competitiva no mercado a mesma necessita de uma avaliação e melhora nos processos. Uma organização necessita de processos avaliados, implementados e reestruturados conforme surgem inovações tecnológicas, agregando valor no mercado e gerando bons resultados positivos para com seus clientes. 


\section{REFERÊNCIAS BIBLIOGRÁFICAS}

ASOFF, H. I.; MCDONNELL, E. Implantando a Administração Estratégica. São Paulo: Atlas, 1993.

BRADY, Malcolm P. Multiple roles of student and instructor in university teaching and learning processes. The International Journal of Management Education, Irlanda, v. 11, n. 2, p. 93-106, 2013.

CAMPOS, A. R.; LIMA, S. M. P. Mapeamento de Processos: Importância para as organizações.Disponível:<http://www.ufrrj.br/codep/materialcursos/projetomapeamento/Map eamentoProcessos>Acesso em: 07 abr. 2018.

DE SORDI, José Osvaldo. Gestão por processos: uma abordagem da moderna administração. 3 ed. rev. e atual. São Paulo: Saraiva, 2012.

FITZSIMMONS, J. A.; FITZSIMMONS, M. J. Administração de serviços: operações, estratégia e tecnologia de informações. Editora Bookman, $2^{\mathrm{a}}$ edição, Porto Alegre, RS, 2000

GEORGE, M. L. Lean Six Sigma for Service: How to Use Lean Speed and Six Sigma Quality to Improve Services and Transactions. New York: The Mcgraw-hill Companies, 2003.

HARRINGTON, H. J. Aperfeiçoando Processos Empresariais. São Paulo: Makron Books, 1993.

MARTINHÃO FILHO, O.; SOUZA, L. G. M. Restrições técnicas associadas a um sistema integrado de gestão: estudo de caso em uma empresa. In: ENCONTRO NACIONAL DE ENGENHARIA DE PRODUÇÃO, 26., 2006. Anais...Fortaleza, CE, 2006.

O'BRIEN, J. et al. Process Papping na analysis of chemicalm pathology services as a tool for quality improvement. Austrália, 1994.

PETENATE, M. Ferramentas para melhoria - SIPOC. Portal Escola Edti, 2012. Disponível em: 〈http://www.escolaedti.com.br/ferramenta-melhoria-sipoc〉. Acesso em: 24 abr. 2018.

RICHARDSON, R. J. Pesquisa social: métodos e técnicas. 3 ed. São Paulo: Atlas, 1999.

SANTOS, R. O Que é Mapeamento de Processo? Disponível em: <http://www.rildosan.com/2011/06/o-que-e-mapeamento-de-processo.html>. Acesso em: 19 abr. 2018.

SLACK, N.; CHAMBERS, S.; JOHNSTON, R. Administração da produção. Tradução: Maria Teresa Corrêa de Oliveira, Fábio Alher. Revisão técnica Henrique Luiz Corrêa. 2 ed. São Paulo: Atlas, 2002.

SWAN, E. SIPOC: A high-level view of a process. Portal online goleansixsigma.com. Disponível em: < https://goleansixsigma.com/sipoc/>. Acesso em: 10/05/2018.

VERGARA, S C. Projetos e Relatórios de Pesquisa em Administração. Atlas, São Paulo, 1998. 